\title{
Some representations of the general solution to a difference equation of additive type
}

Stevo Stević1,2,3*

"Correspondence: sstevic@pttir ${ }^{1}$ Mathematical Institute of the Serbian Academy of Sciences, Beograd, Serbia

2Department of Medical Research, China Medical University Hospital, China Medical University, Taichung, Taiwan, Republic of China Full list of author information is available at the end of the article

\section{Springer}

\section{Abstract}

The general solution to the difference equation

$$
x_{n+1}=\frac{a x_{n} x_{n-1} x_{n-2}+b x_{n-1} x_{n-2}+c x_{n-2}+d}{x_{n} x_{n-1} x_{n-2}}, \quad n \in \mathbb{N}_{0} \text {, }
$$

where $a, b, c \in \mathbb{C}, d \in \mathbb{C} \backslash\{0\}$, is presented by using the coefficients, the initial values $x_{-j}, j=\overline{0,2}$, and the solution to the difference equation

$$
y_{n+1}=a y_{n}+b y_{n-1}+c y_{n-2}+d y_{n-3}, \quad n \in \mathbb{N}_{0},
$$

satisfying the initial conditions $y_{-3}=y_{-2}=y_{-1}=0, y_{0}=1$. The representation complements known ones of the general solutions to the corresponding difference equations of the first and second order. Besides, the general representation formula is investigated in detail and refined by using the roots of the characteristic polynomial

$$
P_{4}(\lambda)=\lambda^{4}-a \lambda^{3}-b \lambda^{2}-c \lambda-d
$$

of the linear equation. The following cases are considered separately: (1) all the roots of the polynomial are distinct; (2) there is a unique double root of the polynomial; (3) there is a triple root of the polynomial and one simple; (4) there is a quadruple root of the polynomial; (5) there are two distinct double roots of the polynomial.

MSC: Primary 39A20; secondary 39A06; 39A45

Keywords: Third-order difference equation; Solvable difference equation; Linear difference equation; Representation of solutions

\section{Introduction}

Regarding some notations which are used in this paper, say that we denote the sets of positive, nonnegative, integer, and complex numbers by $\mathbb{N}, \mathbb{N}, \mathbb{Z}$, and $\mathbb{C}$, respectively. If $l_{1}, l_{2} \in \mathbb{Z}, l_{1} \leq l_{2}$, then the notation $l=\overline{l_{1}, l_{2}}$, which is frequently used throughout the paper, denotes the set of all $l \in \mathbb{Z}$ satisfying the inequalities $l_{1} \leq l \leq l_{2}$. We also use the standard convention $\prod_{j=k}^{k-1} a_{j}=1, k \in \mathbb{Z}$.

(c) The Author(s) 2019. This article is distributed under the terms of the Creative Commons Attribution 4.0 International License (http://creativecommons.org/licenses/by/4.0/), which permits unrestricted use, distribution, and reproduction in any medium, provided you give appropriate credit to the original author(s) and the source, provide a link to the Creative Commons license, and indicate if changes were made. 
A method for solving the linear difference equation

$$
x_{n+k}=a_{k-1} x_{n+k-1}+\cdots+a_{0} x_{n}, \quad n \geq l,
$$

where $k \in \mathbb{N}, l \in \mathbb{Z}, a_{j} \in \mathbb{C}, j=\overline{0, k-1}, a_{0} \neq 0$, has been known to de Moivre yet [1]. See also Euler's book [2] where some old results on solvability can be found. Solvability theory of linear difference equations was developed later, and it became a standard topic of almost any book on difference equations (see, e.g., [3-10]). Equation (1) is practically solvable when $k \leq 4$, whereas if $k>5$ then it is theoretically solvable. These facts are consequences of a well-known form of the general solution to the equation (see, e.g., [3-10]), as well as of the Abel-Ruffini theorem on solvability of the polynomial equations by radicals [11].

Solvability of equation (1) of the corresponding nonhomogeneous linear difference equation, as well as of the nonhomogeneous linear first-order difference equation whose coefficients can be nonconstant, is frequently used in pure and applied mathematics as well as in other branches of science. For some recently studied classes of nonlinear difference equations and systems of nonlinear difference equations which have been solved by using one of these equations, see, e.g., [12-17]. For some recent applications of their solvability, see, e.g., [18-20]. For example, by using solvability methods in [19] and [20], some classes of difference equations are transformed to "integral" forms, which are usually more suitable for studying the existence of bounded or periodic solutions to difference equations than the equations in their original forms.

Employing various changes of variables, many classes of nonlinear difference equations can be transformed to special cases of equation (1), from which solvability of the nonlinear equations can follow.

One of the first of such nonlinear difference equations which appeared in the literature is the following:

$$
x_{n+1}=\frac{\alpha x_{n}+\beta}{\gamma x_{n}+\delta}, \quad n \in \mathbb{N}_{0}
$$

where $\alpha, \beta, \gamma, \delta, z_{0} \in \mathbb{C}$ (the bilinear difference equation).

Namely, if $\alpha \delta-\beta \gamma \neq 0$ and $\gamma \neq 0$ (these conditions eliminate two simple cases), then the change of variables

$$
x_{n}=\frac{y_{n+1}}{\gamma y_{n}}-\frac{\delta}{\gamma}, \quad n \in \mathbb{N}_{0},
$$

transforms equation (2) to

$$
y_{n+2}-(\alpha+\delta) y_{n+1}+(\alpha \delta-\beta \gamma) y_{n}=0, \quad n \in \mathbb{N}_{0},
$$

(see, e.g., [3, 10, 21-25]).

For a method which uses an associated two-dimensional system of difference equations in solving equation (2), see, e.g., [7] or [26]. For some other results on the difference equation and for some applications of the equation, see, e.g., [3, 12, 25, 27-30].

As an explanation for the study in this paper, let us note now that the change of variables

$$
\tilde{x}_{n}=\gamma x_{n}+\delta, \quad n \in \mathbb{N}_{0},
$$


transforms equation (2) to

$$
\tilde{x}_{n+1}=\widetilde{\alpha}+\frac{\widetilde{\beta}}{\widetilde{x}_{n}}, \quad n \in \mathbb{N}_{0},
$$

where

$$
\widetilde{\alpha}=\alpha+\delta \quad \text { and } \quad \tilde{\beta}=\beta \gamma-\alpha \delta \text {, }
$$

which further by the change of variables

$$
\tilde{x}_{n}=\frac{y_{n+1}}{y_{n}}, \quad n \in \mathbb{N}_{0},
$$

where $y_{0}$ is an arbitrary number different from zero, is transformed to equation (4), that is, to

$$
y_{n+2}=\widetilde{\alpha} y_{n+1}+\widetilde{\beta} y_{n}
$$

for $n \in \mathbb{N}_{0}$.

The method for solving equation (5) by using the change of variables (6) is something which is known to any expert. It is much less known that a transformation which transforms equation (7) to equation (5) has been essentially known for more than two centuries and can be found in book [4] by Vincenzo Brunacci. Namely, in nowadays notation, he employed the following change of variables:

$$
y_{n}=A \prod_{j=0}^{n} z_{j}, \quad n \in \mathbb{N}_{0}
$$

from which after some calculation and cancelation, where is tacitly assumed that $z_{n} \neq 0$, $n \in \mathbb{N}_{0}$, equation (7) becomes

$$
z_{n+2} z_{n+1}=\widetilde{\alpha} z_{n+1}+\widetilde{\beta}, \quad n \in \mathbb{N}_{0}
$$

and consequently,

$$
z_{n+2}=\widetilde{\alpha}+\frac{\widetilde{\beta}}{z_{n+1}}, \quad n \in \mathbb{N}_{0}
$$

Note that the last difference equation is equation (5) with indices shifted forward for one.

Remark 1 Note that the change of variables

$$
y_{n}=y_{0} \prod_{j=0}^{n-1} x_{j}, \quad n \in \mathbb{N}_{0} \text {, }
$$

transforms equation (7) to equation (5) directly, and that the change of variables is quite natural. 
Namely, from relation (6), where $\widetilde{x}_{n}$ is replaced by $x_{n}$, it immediately follows that

$$
y_{n}=x_{n-1} y_{n-1}, \quad n \in \mathbb{N},
$$

which is a simple product-type recurrent relation from which relation (9) is easily obtained.

This should be the main reason why Brunacci tried to solve equation (7) by using the change of variables (8). It is also interesting to note that in this way Brunacci "solved" equation (7) in terms of a product of continuous fractions, which is, in fact, a quite complicated representation of general solution to the difference equation.

Now note that the change of variables (8), where $y_{n}$ is replaced by $x_{n}$, transforms equation (1) to the following:

$$
z_{n+k}=a_{k-1}+\frac{a_{k-2}}{z_{n+k-1}}+\cdots+\frac{a_{0}}{\prod_{j=1}^{k-1} z_{n+j}}
$$

for $n \geq l$.

In [25] we explained why the general solutions to some special cases of the difference equations in (2), as well as the general solutions to some special cases of the corresponding two-dimensional systems of difference equations, can be written in terms of the Fibonacci sequence, that is, in terms of the solution to the difference equation

$$
x_{n+1}=x_{n}+x_{n-1}, \quad n \in \mathbb{N},
$$

such that

$$
x_{0}=0 \quad \text { and } \quad x_{1}=1 .
$$

(Many facts related to the Fibonacci sequence and its numerous applications in various branches in mathematics can be found, e.g., in books [31] and [32]; see also [23] and [26] for some basic facts and relations concerning the sequence). Moreover, we have shown that the general solution to equation (2), as well as the general solution to the corresponding two-dimensional system of difference equations, can be written in terms of their coefficients, initial values, and the solution to an associated second-order linear difference equation with constant coefficients satisfying the initial conditions in (11).

Our further investigations on solvability of difference equations and systems of difference equations have shown that these solutions, the solutions to the linear difference equations with constant coefficients of the third order such that $x_{-2}=x_{-1}=0$ and $x_{0}=1$, as well as the solutions to the linear difference equations with constant coefficients of the fourth order such that $x_{-3}=x_{-2}=x_{-1}=0$ and $x_{0}=1$, naturally appear in representations of general solutions to several other classes of difference equations and systems of difference equations. For example, they appear in papers [25, 28-30] predominately connected to the bilinear difference equations, as well as in the representations of general solutions to the product-type difference equations and systems of difference equations, e.g., in [15, 33-39] (see also the references therein). 
For some other results on solvability of difference equations and systems of difference equations, related topics such as invariants for difference equations and systems, and their applications, see, e.g., [3, 5-7, 9, 10, 23, 26, 32, 40-47].

If $k=3$, equation (10) can be written as follows:

$$
x_{n+1}=a+\frac{b}{x_{n}}+\frac{c}{x_{n} x_{n-1}}
$$

for $n \in \mathbb{N}_{0}$.

Employing the change of variables

$$
x_{n}=\frac{y_{n}}{y_{n-1}}, \quad n \geq-1,
$$

it is transformed to

$$
y_{n+1}=a y_{n}+b y_{n-1}+c y_{n-2}, \quad n \in \mathbb{N}_{0},
$$

which is clearly solvable.

Motivated, among other things, by some results in [15, 25, 33-39], recently in [48] we have proved the following theorem extending and theoretically explaining a recent result in the literature.

Theorem 1 Let $a, b \in \mathbb{C}, c \in \mathbb{C} \backslash\{0\}$, and let $\left(t_{n}\right)_{n \geq-2}$ be the solution to equation (14) such that

$$
t_{-2}=t_{-1}=0, \quad t_{0}=1
$$

Then every well-defined solution to equation (12) has the following representation:

$$
x_{n}=\frac{x_{0} x_{-1} t_{n}+x_{-1}\left(t_{n+1}-a t_{n}\right)+c t_{n-1}}{x_{0} x_{-1} t_{n-1}+x_{-1}\left(t_{n}-a t_{n-1}\right)+c t_{n-2}}
$$

for $n \geq-1$.

It is a natural problem to obtain the corresponding representation of the general solution to the following difference equation:

$$
x_{n+1}=a+\frac{b}{x_{n}}+\frac{c}{x_{n} x_{n-1}}+\frac{d}{x_{n} x_{n-1} x_{n-2}}, \quad n \in \mathbb{N}_{0} \text {, }
$$

where $a, b, c \in \mathbb{C}, d \in \mathbb{C} \backslash\{0\}$.

Our aim here is to solve the problem. Besides, we also present more concrete representations of the general solution to equation (17) by using the roots of the polynomial

$$
P_{4}(\lambda)=\lambda^{4}-a \lambda^{3}-b \lambda^{2}-c \lambda-d,
$$

which is associated with a naturally chosen linear difference equation with constant coefficients. 
The following cases are considered separately: (1) all the roots of the polynomial are distinct; (2) there is a unique double root of the polynomial; (3) there is a triple root of the polynomial and one simple; (4) there is a quadruple root of the polynomial; (5) there are two distinct double roots of the polynomial.

\section{Main results}

The main results in this paper are stated and proved in this section.

\subsection{A representation of general solution to equation (17)}

Our first result concerns the problem of representing the general solution to equation (17) in terms of the parameters $a, b, c, d$, initial values, and a specially chosen solution to a linear difference equation with constant coefficients of the fourth order associated with the equation. It complements known representations of the general solutions to the corresponding difference equation of the first order (i.e., of equation (5)), as well as of the second order (i.e., of equation (12)).

Theorem 2 Let $a, b, c \in \mathbb{C}, d \in \mathbb{C} \backslash\{0\}$, and $\left(s_{n}\right)_{n \geq-2}$ be the solution to the equation

$$
y_{n+1}=a y_{n}+b y_{n-1}+c y_{n-2}+d y_{n-3}, \quad n \in \mathbb{N}_{0},
$$

such that

$$
s_{-3}=s_{-2}=s_{-1}=0, \quad s_{0}=1 .
$$

Then every well-defined solution to equation (17) has the following representation:

$$
x_{n}=\frac{s_{n} x_{0} x_{-1} x_{-2}+\left(s_{n+1}-a s_{n}\right) x_{-1} x_{-2}+\left(c s_{n-1}+d s_{n-2}\right) x_{-2}+d s_{n-1}}{s_{n-1} x_{0} x_{-1} x_{-2}+\left(s_{n}-a s_{n-1}\right) x_{-1} x_{-2}+\left(c s_{n-2}+d s_{n-3}\right) x_{-2}+d s_{n-2}}
$$

for $n \geq-2$.

Proof We consider the following four cases: (1) $a \neq 0$, (2) $a=0, b \neq 0$, (3) $a=b=0, c \neq 0$, (4) $a=b=c=0$, separately.

Case $a \neq 0$. The first part of the proof in this case was essentially presented in [49] (see also [36, 38]). Hence, we will present only essential details for the completeness and benefit of the reader.

First note that, by employing (13) in equation (17), it is transformed to equation (19).

Let

$$
\alpha_{1}:=a, \quad \beta_{1}:=b, \quad \gamma_{1}:=c, \quad \delta_{1}:=d .
$$

Employing (19), where $n$ is replaced by $n-2$, in relation (19), where $n$ is replaced by $n-1$, and using (22), we have

$$
\begin{aligned}
y_{n} & =\alpha_{1}\left(a y_{n-2}+b y_{n-3}+c y_{n-4}+d y_{n-5}\right)+\beta_{1} y_{n-2}+\gamma_{1} y_{n-3}+\delta_{1} y_{n-4} \\
& =\alpha_{2} y_{n-2}+\beta_{2} y_{n-3}+\gamma_{2} y_{n-4}+\delta_{2} y_{n-5}
\end{aligned}
$$


for $n \geq 2$, where

$$
\alpha_{2}:=a \alpha_{1}+\beta_{1}, \quad \beta_{2}:=b \alpha_{1}+\gamma_{1}, \quad \gamma_{2}:=c \alpha_{1}+\delta_{1}, \quad \delta_{2}:=d \alpha_{1} .
$$

Assume that for an $l \in \mathbb{N} \backslash\{1\}$ and all $n \geq l$, we have proved

$$
y_{n}=\alpha_{l} y_{n-l}+\beta_{l} y_{n-l-1}+\gamma_{l} y_{n-l-2}+\delta_{l} y_{n-l-3}
$$

and

$$
\alpha_{l}=a \alpha_{l-1}+\beta_{l-1}, \quad \beta_{l}=b \alpha_{l-1}+\gamma_{l-1}, \quad \gamma_{l}=c \alpha_{l-1}+\delta_{l-1}, \quad \delta_{l}=d \alpha_{l-1} .
$$

Employing relation (19) where $n$ is replaced by $n-l-1$ in (25), it follows that

$$
\begin{aligned}
y_{n} & =\alpha_{l}\left(a y_{n-l-1}+b y_{n-l-2}+c y_{n-l-3}+d y_{n-l-4}\right)+\beta_{l} y_{n-l-1}+\gamma_{l} y_{n-l-2}+\delta_{l} y_{n-l-3} \\
& =\alpha_{l+1} y_{n-l-1}+\beta_{l+1} y_{n-l-2}+\gamma_{l+1} y_{n-l-3}+\delta_{l+1} y_{n-l-4}
\end{aligned}
$$

for $n \geq l+1$, where

$$
\alpha_{l+1}:=a \alpha_{l}+\beta_{l}, \quad \beta_{l+1}:=b \alpha_{l}+\gamma_{l}, \quad \gamma_{l+1}:=c \alpha_{l}+\delta_{l}, \quad \delta_{l+1}:=d \alpha_{l} .
$$

Relations (23), (24), (27), (28), and the induction imply that (25) and (26) hold $2 \leq l \leq n$.

Since $d \neq 0$, (26) can be used to calculate $\alpha_{l}, \beta_{l}, \gamma_{l}$, and $\delta_{l}$ for $l \leq 0$. Namely, by taking $l=1,0,-1,-2$, respectively, it easily follows that (see, e.g., $[34,38]$ for some details)

$$
\begin{array}{llll}
\alpha_{0}=1, & \alpha_{-1}=0, & \alpha_{-2}=0, & \alpha_{-3}=0, \\
\beta_{0}=0, & \beta_{-1}=1, & \beta_{-2}=0, & \beta_{-3}=0, \\
\gamma_{0}=0, & \gamma_{-1}=0, & \gamma_{-2}=1, & \gamma_{-3}=0, \\
\delta_{0}=0, & \delta_{-1}=0, & \delta_{-2}=0, & \delta_{-3}=1 .
\end{array}
$$

The relations in (26) further yield

$$
\begin{aligned}
& \alpha_{n}=a \alpha_{n-1}+b \alpha_{n-2}+c \alpha_{n-3}+d \alpha_{n-4}, \\
& \beta_{n}=\alpha_{n+1}-a \alpha_{n}, \\
& \gamma_{n}=c \alpha_{n-1}+d \alpha_{n-2} \\
& \delta_{n}=d \alpha_{n-1}
\end{aligned}
$$

for $n \in \mathbb{Z}$ (since $d \neq 0)$.

If we take $l=n$ in (25), we obtain

$$
y_{n}=\alpha_{n} y_{0}+\beta_{n} y_{-1}+\gamma_{n} y_{-2}+\delta_{n} y_{-3}
$$

for $n \in \mathbb{N}_{0}$, from which along with (31)-(33), we get

$$
y_{n}=\alpha_{n} y_{0}+\left(\alpha_{n+1}-a \alpha_{n}\right) y_{-1}+\left(c \alpha_{n-1}+d \alpha_{n-2}\right) y_{-2}+d \alpha_{n-1} y_{-3}
$$

for $n \in \mathbb{N}_{0}$ (in fact, (35) holds for every $n \in \mathbb{Z}$ ). 
Using (35) in (13), we obtain

$$
x_{n}=\frac{\alpha_{n} y_{0}+\left(\alpha_{n+1}-a \alpha_{n}\right) y_{-1}+\left(c \alpha_{n-1}+d \alpha_{n-2}\right) y_{-2}+d \alpha_{n-1} y_{-3}}{\alpha_{n-1} y_{0}+\left(\alpha_{n}-a \alpha_{n-1}\right) y_{-1}+\left(c \alpha_{n-2}+d \alpha_{n-3}\right) y_{-2}+d \alpha_{n-2} y_{-3}}
$$

for $n \geq-2$, from which, along with (29) and (30), as well as the definition of the sequence $s_{n}$, it follows that

$$
x_{n}=\frac{s_{n} y_{0}+\left(s_{n+1}-a s_{n}\right) y_{-1}+\left(c s_{n-1}+d s_{n-2}\right) y_{-2}+d s_{n-1} y_{-3}}{s_{n-1} y_{0}+\left(s_{n}-a s_{n-1}\right) y_{-1}+\left(c s_{n-2}+d s_{n-3}\right) y_{-2}+d s_{n-2} y_{-3}}
$$

for $n \geq-2$.

Case $a=0, b \neq 0$. Since $a=0$, we have

$$
y_{n+1}=b y_{n-1}+c y_{n-2}+d y_{n-3}, \quad n \in \mathbb{N}_{0} .
$$

Let

$$
\widetilde{\beta}_{1}:=b, \quad \widetilde{\gamma}_{1}:=c, \quad \widetilde{\delta}_{1}:=d, \quad \tilde{\eta}_{1}:=0 .
$$

Employing (37), where $n$ is replaced by $n-3$, in equality (37), where $n$ is replaced by $n-1$, we obtain

$$
\begin{aligned}
y_{n} & =\widetilde{\beta}_{1}\left(b y_{n-4}+c y_{n-5}+d y_{n-6}\right)+\widetilde{\gamma}_{1} y_{n-3}+\widetilde{\delta}_{1} y_{n-4}+\widetilde{\eta}_{1} y_{n-5} \\
& =\widetilde{\gamma}_{1} y_{n-3}+\left(b \widetilde{\beta}_{1}+\widetilde{\delta}_{1}\right) y_{n-4}+\left(c \widetilde{\beta}_{1}+\widetilde{\eta}_{1}\right) y_{n-5}+d \widetilde{\beta}_{1} y_{n-6} \\
& =\widetilde{\beta}_{2} y_{n-3}+\widetilde{\gamma}_{2} y_{n-4}+\widetilde{\delta}_{2} y_{n-5}+\widetilde{\eta}_{2} y_{n-6}
\end{aligned}
$$

for $n \geq 3$, where

$$
\widetilde{\beta}_{2}:=\widetilde{\gamma}_{1}, \quad \widetilde{\gamma}_{2}:=b \widetilde{\beta}_{1}+\widetilde{\delta}_{1}, \quad \widetilde{\delta}_{2}:=c \widetilde{\beta}_{1}+\widetilde{\eta}_{1}, \quad \widetilde{\eta}_{2}:=d \widetilde{\beta}_{1} .
$$

Assume that for an $l \in \mathbb{N} \backslash\{1\}$ and all $n \geq l+1$, we have proved

$$
y_{n}=\widetilde{\beta}_{l} y_{n-l-1}+\tilde{\gamma}_{l} y_{n-l-2}+\tilde{\delta}_{l} y_{n-l-3}+\tilde{\eta}_{l} y_{n-l-4}
$$

and

$$
\widetilde{\beta}_{l}=\tilde{\gamma}_{l-1}, \quad \tilde{\gamma}_{l}=b \widetilde{\beta}_{l-1}+\widetilde{\delta}_{l-1}, \quad \widetilde{\delta}_{l}=c \widetilde{\beta}_{l-1}+\tilde{\eta}_{l-1}, \quad \tilde{\eta}_{l}=d \widetilde{\beta}_{l-1} .
$$

Then, by using equality (37) where $n$ is replaced by $n-l-2$ in (41), we have

$$
\begin{aligned}
y_{n} & =\widetilde{\beta}_{l}\left(b y_{n-l-3}+c y_{n-l-4}+d y_{n-l-5}\right)+\tilde{\gamma}_{l} y_{n-l-2}+\widetilde{\delta}_{l} y_{n-l-3}+\tilde{\eta}_{l} y_{n-l-4} \\
& =\widetilde{\gamma}_{l} y_{n-l-2}+\left(b \widetilde{\beta}_{l}+\widetilde{\delta}_{l}\right) y_{n-l-3}+\left(c \widetilde{\beta}_{l}+\widetilde{\eta}_{l}\right) y_{n-l-4}+d \widetilde{\beta}_{l} y_{n-l-5} \\
& =\widetilde{\beta}_{l+1} y_{n-l-2}+\widetilde{\gamma}_{l+1} y_{n-l-3}+\widetilde{\delta}_{l+1} y_{n-l-4}+\widetilde{\eta}_{l+1} y_{n-l-5},
\end{aligned}
$$


where

$$
\widetilde{\beta}_{l+1}:=\widetilde{\gamma}_{l}, \quad \widetilde{\gamma}_{l+1}:=b \widetilde{\beta}_{l}+\widetilde{\delta}_{l}, \quad \widetilde{\delta}_{l+1}:=c \widetilde{\beta}_{l}+\widetilde{\eta}_{l}, \quad \tilde{\eta}_{l+1}:=d \widetilde{\beta}_{l} .
$$

Relations (39), (40), (43), (44) together with the induction imply that (41) and (42) hold for $l, n \in \mathbb{N}$ such that $2 \leq l \leq n-1$.

Since $d \neq 0$, from (42) it follows that $\widetilde{\beta}_{l}, \widetilde{\gamma}_{l}, \widetilde{\delta}_{l}, \widetilde{\eta}_{l}$ can be calculated for $l \leq 0$. Namely, by taking $l=1,0,-1,-2,-3$, respectively, it easily follows that

$$
\begin{array}{llll}
\tilde{\beta}_{-1}=1, & \tilde{\beta}_{-2}=0, & \tilde{\beta}_{-3}=0, & \widetilde{\beta}_{-4}=0, \\
\tilde{\gamma}_{-1}=0, & \tilde{\gamma}_{-2}=1, & \tilde{\gamma}_{-3}=0, & \tilde{\gamma}_{-4}=0, \\
\widetilde{\delta}_{-1}=0, & \widetilde{\delta}_{-2}=0, & \tilde{\delta}_{-3}=1, & \widetilde{\delta}_{-4}=0, \\
\tilde{\eta}_{-1}=0, & \tilde{\eta}_{-2}=0, & \tilde{\eta}_{-3}=0, & \tilde{\eta}_{-4}=1,
\end{array}
$$

(for $l=1$ are obtained $\widetilde{\beta}_{0}, \widetilde{\gamma}_{0}, \widetilde{\delta}_{0}, \widetilde{\eta}_{0}$, but they are not used further, which is why we have not listed them here).

The relations in (42) further yield

$$
\begin{aligned}
& \widetilde{\beta}_{n}=b \widetilde{\beta}_{n-2}+c \widetilde{\beta}_{n-3}+d \widetilde{\beta}_{n-4}, \\
& \widetilde{\gamma}_{n}=\widetilde{\beta}_{n+1}, \\
& \widetilde{\delta}_{n}=c \widetilde{\beta}_{n-1}+d \widetilde{\beta}_{n-2} \\
& \widetilde{\eta}_{n}=d \widetilde{\beta}_{n-1}
\end{aligned}
$$

for $n \in \mathbb{Z}$.

From (41) with $l=n-1$, we obtain

$$
y_{n}=\widetilde{\beta}_{n-1} y_{0}+\widetilde{\gamma}_{n-1} y_{-1}+\widetilde{\delta}_{n-1} y_{-2}+\widetilde{\eta}_{n-1} y_{-3}
$$

for $n \in \mathbb{N}_{0}$.

Employing (47)-(49) in (50), we get

$$
y_{n}=\widetilde{\beta}_{n-1} y_{0}+\widetilde{\beta}_{n} y_{-1}+\left(c \widetilde{\beta}_{n-2}+d \widetilde{\beta}_{n-3}\right) y_{-2}+d \widetilde{\beta}_{n-2} y_{-3}
$$

for $n \in \mathbb{N}_{0}$

From (45) and (46) it follows that $\widetilde{\beta}_{n}$ is the solution to equation (19) with $a=0, b \neq 0$ such that

$$
\widetilde{\beta}_{n-1}=s_{n} .
$$

Hence, from (13) and (51) it follows that (36) also holds in this case.

Case $a=b=0 \neq c$. Since $a=b=0$, equation (19) is

$$
y_{n+1}=c y_{n-2}+d y_{n-3}
$$

for $n \in \mathbb{N}_{0}$. 
Let

$$
\widehat{\gamma}_{1}:=c, \quad \widehat{\delta}_{1}:=d, \quad \widehat{\eta}_{1}:=0, \quad \widehat{\zeta}_{1}:=0
$$

Employing (52), where $n$ is replaced by $n-4$, in equality (52), where $n$ is replaced by $n-1$, we obtain

$$
\begin{aligned}
y_{n} & =\widehat{\gamma}_{1} y_{n-3}+\widehat{\delta}_{1} y_{n-4}+\widehat{\eta}_{1} y_{n-5}+\widehat{\zeta}_{1} y_{n-6} \\
& =\widehat{\gamma}_{1}\left(c y_{n-6}+d y_{n-7}\right)+\widehat{\delta}_{1} y_{n-4}+\widehat{\eta}_{1} y_{n-5}+\widehat{\zeta}_{1} y_{n-6} \\
& =\widehat{\delta}_{1} y_{n-4}+\widehat{\eta}_{1} y_{n-5}+\left(c \widehat{\gamma}_{1}+\widehat{\zeta}_{1}\right) y_{n-6}+d \widehat{\gamma}_{1} y_{n-7} \\
& =\widehat{\gamma}_{2} y_{n-4}+\widehat{\delta}_{2} y_{n-5}+\widehat{\eta}_{2} y_{n-6}+\widehat{\zeta}_{2} y_{n-7}
\end{aligned}
$$

for $n \geq 4$, where

$$
\widehat{\gamma}_{2}:=\widehat{\delta}_{1}, \quad \widehat{\delta}_{2}:=\widehat{\eta}_{1}, \quad \widehat{\eta}_{2}:=c \widehat{\gamma}_{1}+\widehat{\zeta}_{1}, \quad \widehat{\zeta}_{2}:=d \widehat{\gamma}_{1}
$$

Assume that for an $l \in \mathbb{N} \backslash\{1\}$ and all $n \geq l+2$, we have proved

$$
y_{n}=\widehat{\gamma}_{l} y_{n-l-2}+\widehat{\delta}_{l} y_{n-l-3}+\widehat{\eta}_{l} y_{n-l-4}+\widehat{\zeta}_{l} y_{n-l-5}
$$

and

$$
\widehat{\gamma}_{l}=\widehat{\delta}_{l-1}, \quad \widehat{\delta}_{l}=\widehat{\eta}_{l-1}, \quad \widehat{\eta}_{l}=c \widehat{\gamma}_{l-1}+\widehat{\zeta}_{l-1}, \quad \widehat{\zeta}_{l}=d \widehat{\gamma} l-1
$$

Then, by using equality (52) where $n$ is replaced by $n-l-3$ in (56), we have

$$
\begin{aligned}
y_{n} & =\widehat{\gamma}_{l} y_{n-l-2}+\widehat{\delta}_{l} y_{n-l-3}+\widehat{\eta}_{l} y_{n-l-4}+\widehat{\zeta}_{l} y_{n-l-5} \\
& =\widehat{\gamma}_{l}\left(c y_{n-l-5}+d y_{n-l-6}\right)+\widehat{\delta}_{l} y_{n-l-3}+\widehat{\eta}_{l} y_{n-l-4}+\widehat{\zeta} y_{n-l-5} \\
& =\widehat{\delta}_{l} y_{n-l-3}+\widehat{\eta}_{l} y_{n-l-4}+\left(c \widehat{\gamma}_{l}+\widehat{\zeta}_{l}\right) y_{n-l-5}+d \widehat{\gamma}_{l} y_{n-l-6} \\
& =\widehat{\gamma}_{l+1} y_{n-l-3}+\widehat{\delta}_{l+1} y_{n-l-4}+\widehat{\eta}_{l+1} y_{n-l-5}+\widehat{\zeta}_{l+1} y_{n-l-6},
\end{aligned}
$$

where

$$
\widehat{\gamma}_{l+1}:=\widehat{\delta}_{l}, \quad \widehat{\delta}_{l+1}:=\widehat{\eta}_{l}, \quad \widehat{\eta}_{l+1}:=c \widehat{\gamma}_{l}+\widehat{\zeta}_{l}, \quad \widehat{\zeta}_{l+1}:=d \widehat{\gamma}_{l}
$$

From (54), (55), (58), (59), and the induction it follows that (56) and (57) hold for $l, n \in \mathbb{N}$ such that $2 \leq l \leq n-2$.

Since $d \neq 0$, employing the recurrent relations (57), we can calculate $\widehat{\gamma}_{l}, \widehat{\delta}_{l}, \widehat{\eta}_{l}, \widehat{\zeta}_{l}$ for $l \leq 0$ and get

$$
\begin{array}{llll}
\widehat{\gamma}_{-2}=1, & \widehat{\gamma}_{-3}=0, & \widehat{\gamma}_{-4}=0, & \widehat{\gamma}_{-5}=0, \\
\widehat{\delta}_{-2}=0, & \widehat{\delta}_{-3}=1, & \widehat{\delta}_{-4}=0, & \widehat{\delta}_{-5}=0, \\
\widehat{\eta}_{-2}=0, & \widehat{\eta}_{-3}=0, & \widehat{\eta}_{-4}=1, & \widehat{\eta}_{-5}=0, \\
\widehat{\zeta}_{-2}=0, & \widehat{\zeta}_{-3}=0, & \widehat{\zeta}_{-4}=0, & \widehat{\zeta}_{-5}=1
\end{array}
$$

(see, e.g., the corresponding calculations in [34, 37]). 
The relations in (57) further yield

$$
\begin{aligned}
& \widehat{\gamma}_{n}=c \widehat{\gamma}_{n-3}+d \widehat{\gamma}_{n-4}, \\
& \widehat{\delta}_{n}=\widehat{\gamma}_{n+1}, \\
& \widehat{\eta}_{n}=c \widehat{\gamma}_{n-1}+d \widehat{\gamma}_{n-2}, \\
& \widehat{\zeta}_{n}=d \widehat{\gamma}_{n-1},
\end{aligned}
$$

for $n \in \mathbb{Z}$.

If we choose $l=n-2$ in (56), we obtain

$$
y_{n}=\widehat{\gamma}_{n-2} y_{0}+\widehat{\delta}_{n-2} y_{-1}+\widehat{\eta}_{n-2} y_{-2}+\widehat{\zeta}_{n-2} y_{-3}
$$

for $n \in \mathbb{N}_{0}$.

Employing (62)-(64) in (65), it follows that

$$
y_{n}=\widehat{\gamma}_{n-2} y_{0}+\widehat{\gamma}_{n-1} y_{-1}+\left(c \widehat{\gamma}_{n-3}+d \widehat{\gamma}_{n-4}\right) y_{-2}+d \widehat{\gamma}_{n-3} y_{-3}
$$

for $n \in \mathbb{N}_{0}$.

From (60) and (61) we see that $\widehat{\gamma}_{n}$ is the solution to equation (19) with $a=b=0, c \neq 0$ such that

$$
\widehat{\gamma}_{n-2}=s_{n},
$$

from which along with (13) and (65) it follows that (36) also holds in this case.

Case $a=b=c=0 \neq d$. Since $a=b=c=0$, equation (19) is

$$
y_{n+1}=d y_{n-3}
$$

for $n \in \mathbb{N}_{0}$.

From (67) we have

$$
s_{4 m-i}=d^{m} s_{-i}
$$

for $m \in \mathbb{N}_{0}$ and $i=\overline{0,3}$. It is not difficult to check that (36) holds for the sequence defined in (68).

From (36) we have

$$
\begin{aligned}
x_{n} & =\frac{s_{n} y_{0}+\left(s_{n+1}-a s_{n}\right) y_{-1}+\left(c s_{n-1}+d s_{n-2}\right) y_{-2}+d s_{n-1} y_{-3}}{s_{n-1} y_{0}+\left(s_{n}-a s_{n-1}\right) y_{-1}+\left(c s_{n-2}+d s_{n-3}\right) y_{-2}+d s_{n-2} y_{-3}} \\
& =\frac{s_{n} \frac{y_{0}}{y_{-3}}+\left(s_{n+1}-a s_{n}\right) \frac{y_{-1}}{y_{-3}}+\left(c s_{n-1}+d s_{n-2}\right) \frac{y_{-2}}{y_{-3}}+d s_{n-1}}{s_{n-1} \frac{y_{0}}{y_{-3}}+\left(s_{n}-a s_{n-1}\right) \frac{y_{-1}}{y_{-3}}+\left(c s_{n-2}+d s_{n-3}\right) \frac{y_{-2}}{y_{-3}}+d s_{n-2}} \\
& =\frac{s_{n} x_{0} x_{-1} x_{-2}+\left(s_{n+1}-a s_{n}\right) x_{-1} x_{-2}+\left(c s_{n-1}+d s_{n-2}\right) x_{-2}+d s_{n-1}}{s_{n-1} x_{0} x_{-1} x_{-2}+\left(s_{n}-a s_{n-1}\right) x_{-1} x_{-2}+\left(c s_{n-2}+d s_{n-3}\right) x_{-2}+d s_{n-2}}
\end{aligned}
$$

for $n \in \mathbb{Z}$, as claimed. 
Remark 2 The case $a \neq 0$ has been essentially treated in [49]. However, the proofs in the other cases, in all of which is $a=0$, have been omitted there; although, as it is seen in the proof of Theorem 2, they follow the idea of the proof in the case $a \neq 0$. Hence, the proof of Theorem 2 can be regarded as a completion of the proof of Theorem 6 in [49] in the case $a=0$.

\subsection{About the roots of polynomial $P_{4}(\lambda)$}

Here we present the roots of polynomial $P_{4}$ and some facts related to them.

First, we formulate a well-known lemma, whose proofs can be found, e.g., in [26, 38, 50].

Lemma 1 Let $t_{j}, j=\overline{1, k}$ be the roots of the polynomial

$$
p(t)=\widetilde{a}_{k} t^{k}+\widetilde{a}_{k-1} t^{k-1}+\cdots+\widetilde{a}_{1} t+\widetilde{a}_{0}
$$

such that $t_{i} \neq t_{j}, i \neq j$, then

$$
\sum_{j=1}^{k} \frac{t_{j}^{s}}{p^{\prime}\left(t_{j}\right)}= \begin{cases}0, & s=\overline{0, k-2} \\ 1 / \widetilde{a}_{k}, & s=k-1 .\end{cases}
$$

Now we discuss the form of the sequence $s_{n}$ defined in Theorem 2. Equation $P_{4}(\lambda)=0$ can be written in the form

$$
\left(\lambda^{2}-\frac{a}{2} \lambda+\frac{s}{2}\right)^{2}-\left(\left(\frac{a^{2}}{4}+b+s\right) \lambda^{2}-\left(\frac{a s}{2}-c\right) \lambda+\frac{s^{2}}{4}+d\right)=0
$$

Further, choose $s$ so that

$$
(a s-2 c)^{2}=\left(a^{2}+4 b+4 s\right)\left(s^{2}+4 d\right)
$$

(see, for example, [50]), that is,

$$
s^{3}+b s^{2}+(a c+4 d) s+a^{2} d+4 b d-c^{2}=0 .
$$

Then (69) is

$$
\left(\lambda^{2}-\frac{a}{2} \lambda+\frac{s}{2}\right)^{2}-\left(\frac{\sqrt{a^{2}+4 b+4 s}}{2} \lambda-\frac{a s-2 c}{2 \sqrt{a^{2}+4 b+4 s}}\right)^{2}=0
$$

from which it follows that

$$
\lambda^{2}-\left(\frac{a}{2}+\frac{\sqrt{a^{2}+4 b+4 s}}{2}\right) \lambda+\frac{s}{2}+\frac{a s-2 c}{2 \sqrt{a^{2}+4 b+4 s}}=0
$$

or

$$
\lambda^{2}-\left(\frac{a}{2}-\frac{\sqrt{a^{2}+4 b+4 s}}{2}\right) \lambda+\frac{s}{2}-\frac{a s-2 c}{2 \sqrt{a^{2}+4 b+4 s}}=0 .
$$


By using the change of variables

$$
s=\widehat{s}-\frac{b}{3}
$$

in (70), we have

$$
\widehat{s}^{3}+\widehat{p s}+q=0,
$$

where

$$
p=a c+4 d-\frac{b^{2}}{3}
$$

and

$$
q=a^{2} d+\frac{8 b d}{3}-\frac{a b c}{3}-c^{2}+\frac{2 b^{3}}{27} .
$$

A standard procedure for solving cubic equation (75) says that the solutions should be looked for in the form $\widehat{s}=u+v$, where it is assumed that $u v=-p / 3$. Hence, $u^{3}+v^{3}=-q$ and $u^{3} v^{3}=-p^{3} / 27$, implying that $u^{3}$ and $v^{3}$ are the zeros of $z^{2}+q z-p^{3} / 27=0$.

Thus

$$
\widehat{s}=\sqrt[3]{-\frac{q}{2}-\sqrt{\frac{q^{2}}{4}+\frac{p^{3}}{27}}}+\sqrt[3]{-\frac{q}{2}+\sqrt{\frac{q^{2}}{4}+\frac{p^{3}}{27}}}
$$

Let $p=-\Delta_{0} / 3$ and $q=-\Delta_{1} / 27$, then from (74) and (76) we have

$$
s=\frac{1}{3 \sqrt[3]{2}}\left(\sqrt[3]{\Delta_{1}-\sqrt{\Delta_{1}^{2}-4 \Delta_{0}^{3}}}+\sqrt[3]{\Delta_{1}+\sqrt{\Delta_{1}^{2}-4 \Delta_{0}^{3}}}\right)-\frac{b}{3} .
$$

From (72) and (73) and by some calculations, we obtain

$$
\begin{aligned}
& \lambda_{1}=\frac{a}{4}+\frac{\sqrt{a^{2}+4 b+4 s}}{4}+\frac{1}{2} \sqrt{\frac{a^{2}}{2}+b-s-\frac{Q}{2 \sqrt{a^{2}+4 b+4 s}},} \\
& \lambda_{2}=\frac{a}{4}+\frac{\sqrt{a^{2}+4 b+4 s}}{4}-\frac{1}{2} \sqrt{\frac{a^{2}}{2}+b-s-\frac{Q}{2 \sqrt{a^{2}+4 b+4 s}}}, \\
& \lambda_{3}=\frac{a}{4}-\frac{\sqrt{a^{2}+4 b+4 s}}{4}+\frac{1}{2} \sqrt{\frac{a^{2}}{2}-s+\frac{Q}{2 \sqrt{a^{2}+4 b+4 s}},} \\
& \lambda_{4}=\frac{a}{4}-\frac{\sqrt{a^{2}+4 b+4 s}}{4}-\frac{1}{2} \sqrt{\frac{a^{2}}{2}+b-s+\frac{Q}{2 \sqrt{a^{2}+4 b+4 s}}},
\end{aligned}
$$

where

$$
\begin{aligned}
& \Delta_{0}=-3 p=b^{2}-3 a c-12 d, \\
& \Delta_{1}=-27 q=-2 b^{3}-27 a^{2} d-72 b d+9 a b c+27 c^{2}, \\
& Q=-a^{3}-4 a b-8 c .
\end{aligned}
$$


Multiplicity of the zeros of the polynomial $P_{4}$ are described in the following lemma (see, e.g., [51]).

Lemma 2 Let polynomial $P_{4}$ be defined in (18), $\Delta_{0}$ in (82), $\Delta_{1}$ in (83), $Q$ in (84), and let $\Delta=\frac{1}{27}\left(4 \Delta_{0}^{3}-\Delta_{1}^{2}\right), P=-8 b-3 a^{2}$, and $D=-64 d-16 b^{2}-16 a^{2} b-16 a c-3 a^{4}$.

Then the following statements hold:

(a) If $\Delta \neq 0$, then the zeros of $P_{4}$ are distinct.

(b) If $\Delta=0$ and $P<0, D<0, \Delta_{0} \neq 0$, or $D>0$, or $P>0, D \neq 0$, or $P>0, Q \neq 0$, then exactly two zeros of $P_{4}$ are equal.

(c) If $\Delta=0, \Delta_{0}=0$, and $D \neq 0$, then there is a triple zero of $P_{4}$ and one simple.

(d) If $\Delta=0, D=0$, and if $P<0$, or $P>0$ and $Q=0$, then $P_{4}$ has two distinct double zeros.

(e) If $\Delta=0, D=0$, and $\Delta_{0}=0$, then all the zeros of $P_{4}$ are equal to $a / 4$.

\subsection{Possible forms of the sequence $s_{n}$}

While formula (21) gives a representation of the general solution to equation (17) in terms of the sequence $s_{n}$, it does not tell anything about its possible forms. In this section we will describe the forms in terms of the roots $\lambda_{j}, j=\overline{1,4}$, of polynomial (18).

Case $\Delta \neq 0$. In this case, by Lemma 2(a), we have that the roots $\lambda_{j}, j=\overline{1,4}$, are distinct. By using Lemma 1 and the general solution to equation (19) in this case, we have proved in [38] (see also [36]) that the following formula holds:

$$
s_{n}=\sum_{j=1}^{4} \frac{\lambda_{j}^{n+3}}{P_{4}^{\prime}\left(\lambda_{j}\right)}
$$

for $n \geq-3$ (in fact, (85) holds for every $n \in \mathbb{Z}$ ).

If one of the roots of $P_{4}$ is one, say $\lambda_{1}$, then we have

$$
P_{4}(\lambda)=(\lambda-1)\left(\lambda^{3}+(1-a) \lambda^{2}+(1-a-b) \lambda+1-a-b-c\right) .
$$

Employing the change of variables

$$
\lambda=s-\frac{1-a}{3}
$$

in the equation

$$
\lambda^{3}+(1-a) \lambda^{2}+(1-a-b) \lambda+1-a-b-c=0
$$

it is transformed to

$$
s^{3}+\widehat{p} s+\widehat{q}=0
$$

where

$$
\widehat{p}=1-a-b-\frac{(1-a)^{2}}{3} \text { and } \widehat{q}=\frac{2(1-a)^{3}}{27}+\frac{(1-a-b)(2+a)}{3}-c \text {. }
$$


We have (see [50]; see also (76))

$$
s_{j}=\sqrt[3]{-\frac{\widehat{q}}{2}-\sqrt{\frac{\widehat{q}^{2}}{4}+\frac{\widehat{p}^{3}}{27}}} \varepsilon^{j-2}+\sqrt[3]{-\frac{\widehat{q}}{2}+\sqrt{\frac{\widehat{q}^{2}}{4}+\frac{\widehat{p}^{3}}{27}}} \bar{\varepsilon}^{j-2}
$$

for $j=\overline{2,4}$, where $\varepsilon$ is a complex root of the equation $t^{3}=1$, from which along with (87) it follows that

$$
\lambda_{j}=\sqrt[3]{-\frac{\widehat{q}}{2}-\sqrt{\frac{\widehat{q}^{2}}{4}+\frac{\widehat{p}^{3}}{27}}} \varepsilon^{j-2}+\sqrt[3]{-\frac{\widehat{q}}{2}+\sqrt{\frac{\widehat{q}^{2}}{4}+\frac{\widehat{p}^{3}}{27}}} \bar{\varepsilon}^{j-2}-\frac{1-a}{3}
$$

for $j=\overline{2,4}$.

From the above analysis, we get the following corollary.

Corollary 1 Consider equation (17) with $a, b, c \in \mathbb{C}$ and $d \in \mathbb{C} \backslash\{0\}$. Assume that $\Delta \neq 0$. Then the following statements are true.

(a) If $a+b+c+d \neq 1$, then the general solution to equation (17) is given by (21), where sequence $\left(s_{n}\right)_{n \geq-3}$ is given by (85), whereas $\lambda_{j}, j=\overline{1,4}$, are given by (78)-(81).

(b) If $a+b+c+d=1$, then the general solution to equation (17) is given by (21), where sequence $\left(s_{n}\right)_{n \geq-3}$ is given by (85) where $\lambda_{1}=1, \lambda_{j}, j=\overline{2,4}$, are given by (89).

Remark 3 Note that from (85) we have

$$
\begin{aligned}
& s_{n+1}-a s_{n}=\sum_{j=1}^{4} \frac{\lambda_{j}-a}{P_{4}^{\prime}\left(\lambda_{j}\right)} \lambda_{j}^{n+3}, \\
& c s_{n-1}+d s_{n-2}=\sum_{j=1}^{4} \frac{c \lambda_{j}+d}{P_{4}^{\prime}\left(\lambda_{j}\right)} \lambda_{j}^{n+1}, \\
& d s_{n-1}=\sum_{j=1}^{4} \frac{d}{P_{4}^{\prime}\left(\lambda_{j}\right)} \lambda_{j}^{n+2}
\end{aligned}
$$

for $n \in \mathbb{Z}$. By using (85), (90)-(92) in (21), we get the following representation of the general solution to equation (17) in the case $\Delta \neq 0$ :

$$
x_{n}=\frac{\sum_{j=1}^{4} \frac{\lambda_{j}^{2} x_{0} x_{-1} x_{-2}+\left(\lambda_{j}^{3}-a \lambda_{j}^{2}\right) x_{-1} x_{-2}+\left(c \lambda_{j}+d\right) x_{-2}+d \lambda_{j}}{P_{4}^{\prime}\left(\lambda_{j}\right)} \lambda_{j}^{n+1}}{\sum_{j=1}^{4} \frac{\lambda_{j}^{2} x_{0} x_{-1} x_{-2}+\left(\lambda_{j}^{3}-a \lambda_{j}^{2}\right) x_{-1} x_{-2}+\left(c \lambda_{j}+d\right) x_{-2}+d \lambda_{j}}{P_{4}^{\prime}\left(\lambda_{j}\right)} \lambda_{j}^{n}}
$$

for $n \in \mathbb{Z}$

Case $\Delta=0$ and $P<0, D<0, \Delta_{0} \neq 0$, or $D>0$, or $P>0, D \neq 0$, or $P>0, Q \neq 0$. By Lemma 2(b) in this case $P_{4}$ has exactly one double zero. We may assume $\lambda_{1}=\lambda_{2} \neq \lambda_{j}$, 
$j=3,4$. To find the solution satisfying (20), we let $\lambda_{1} \rightarrow \lambda_{2}$ in (85) and get

$$
\begin{aligned}
s_{n}= & \frac{\lambda_{2}^{n+2}\left((n+3)\left(\lambda_{2}-\lambda_{3}\right)\left(\lambda_{2}-\lambda_{4}\right)-\lambda_{2}\left(2 \lambda_{2}-\lambda_{3}-\lambda_{4}\right)\right)}{\left(\lambda_{2}-\lambda_{3}\right)^{2}\left(\lambda_{2}-\lambda_{4}\right)^{2}} \\
& +\frac{\lambda_{3}^{n+3}}{\left(\lambda_{3}-\lambda_{2}\right)^{2}\left(\lambda_{3}-\lambda_{4}\right)}+\frac{\lambda_{4}^{n+3}}{\left(\lambda_{4}-\lambda_{2}\right)^{2}\left(\lambda_{4}-\lambda_{3}\right)}
\end{aligned}
$$

for $n \in \mathbb{Z}$ (for details, see [36]).

If $\lambda_{1}=\lambda_{2}=1$, then from (94) we immediately get

$$
\begin{aligned}
s_{n}= & \frac{n\left(1-\lambda_{3}\right)\left(1-\lambda_{4}\right)+3 \lambda_{3} \lambda_{4}-2 \lambda_{3}-2 \lambda_{4}+1}{\left(1-\lambda_{3}\right)^{2}\left(1-\lambda_{4}\right)^{2}} \\
& +\frac{\lambda_{3}^{n+3}}{\left(\lambda_{3}-1\right)^{2}\left(\lambda_{3}-\lambda_{4}\right)}+\frac{\lambda_{4}^{n+3}}{\left(\lambda_{4}-1\right)^{2}\left(\lambda_{4}-\lambda_{3}\right)}
\end{aligned}
$$

for $n \in \mathbb{Z}$.

In this case we have $a+b+c+d-1=4-3 a-2 b-c=0,12 \neq 6 a+2 b$, and

$$
P_{4}(\lambda)=(\lambda-1)^{2}\left(\lambda^{2}+(2-a) \lambda+3-2 a-b\right),
$$

from which it follows that

$$
\lambda_{3,4}=\frac{a-2 \pm \sqrt{a^{2}+4 a+4 b-8}}{2} .
$$

Corollary 2 Consider equation (17) with $a, b, c \in \mathbb{C}$ and $d \in \mathbb{C} \backslash\{0\}$. Assume that $\Delta=0$ and $P<0, D<0, \Delta_{0} \neq 0$, or $D>0$, or $P>0, D \neq 0$, or $P>0, Q \neq 0$. Then the following statements hold.

(a) If $3 a+2 b+c \neq 4$, then the general solution to equation (17) is given by formula (21), where sequence $\left(s_{n}\right)_{n \geq-3}$ is given by (94).

(b) If $a+b+c+d-1=4-3 a-2 b-c=0$, then the general solution to equation (17) is given by formula (21), where sequence $\left(s_{n}\right)_{n \geq-3}$ is given by (95), whereas $\lambda_{3,4}$ are given by (97).

Case $\Delta=0, D=0$, and $P<0$, or $P>0$ and $Q=0$. By Lemma $2(\mathrm{~d})$ in this case $P_{4}$ has two pairs of double zeros. By letting $\lambda_{3} \rightarrow \lambda_{4}$ in (94), it is obtained

$$
\begin{aligned}
s_{n}= & \frac{\lambda_{2}^{n+2}\left(n\left(\lambda_{2}-\lambda_{4}\right)^{2}+\lambda_{2}^{2}-4 \lambda_{2} \lambda_{4}+3 \lambda_{4}^{2}\right)}{\left(\lambda_{2}-\lambda_{4}\right)^{4}} \\
& +\frac{\lambda_{4}^{n+2}\left(n\left(\lambda_{4}-\lambda_{2}\right)^{2}+\lambda_{4}^{2}-4 \lambda_{2} \lambda_{4}+3 \lambda_{2}^{2}\right)}{\left(\lambda_{4}-\lambda_{2}\right)^{4}}
\end{aligned}
$$

for $n \geq-3$ (see [36]).

If $a+b+c+d=1$, then two of the zeros, say $\lambda_{1}$ and $\lambda_{2}$, are equal to 1 , and from (98) we obtain

$$
s_{n}=\frac{\lambda_{4}^{n+2}\left(n\left(\lambda_{4}-1\right)^{2}+\lambda_{4}^{2}-4 \lambda_{4}+3\right)+n\left(1-\lambda_{4}\right)^{2}+1-4 \lambda_{4}+3 \lambda_{4}^{2}}{\left(\lambda_{4}-1\right)^{4}}
$$


for $n \geq-3$. Besides, from (97) we have

$$
\lambda_{3,4}=\frac{a-2}{2} .
$$

Corollary 3 Consider equation (17) with $a, b, c \in \mathbb{C}$ and $d \in \mathbb{C} \backslash\{0\}$. Assume that $\Delta=0$, $D=0$, and $P<0$, or $P>0$ and $Q=0$. Then the following statements hold.

(a) If $a+b+c+d \neq 1$, then the general solution to equation (17) is given by formula (21), where sequence $\left(s_{n}\right)_{n \geq-3}$ is given by (98).

(b) If $a+b+c+d=1$, then the general solution to equation (17) is given by formula (21), where sequence $\left(s_{n}\right)_{n \geq-3}$ is given by (99), whereas $\lambda_{3,4}$ are given by (100).

Case $\Delta=0, \Delta_{0}=0$, and $D \neq 0$. By Lemma 2(c) we have that $P_{4}$ has a triple zero and one simple. We may assume that $\lambda_{1}=\lambda_{2}=\lambda_{3}$. In [35] we proved that in this case

$$
s_{n}=\left(1-\frac{\lambda_{4}^{3}}{\left(\lambda_{4}-\lambda_{1}\right)^{3}}+\frac{\lambda_{1}\left(3 \lambda_{1}-5 \lambda_{4}\right)}{2\left(\lambda_{4}-\lambda_{1}\right)^{2}} n+\frac{\lambda_{1}}{2\left(\lambda_{1}-\lambda_{4}\right)} n^{2}\right) \lambda_{1}^{n}+\frac{\lambda_{4}^{n+3}}{\left(\lambda_{4}-\lambda_{1}\right)^{3}}
$$

for $n \geq-3$.

$$
\text { If } \lambda_{j}=1, j=\overline{1,3} \text {, then from (101) it follows that }
$$

$$
s_{n}=1-\frac{\lambda_{4}^{3}}{\left(\lambda_{4}-1\right)^{3}}+\frac{\left(3-5 \lambda_{4}\right)}{2\left(\lambda_{4}-1\right)^{2}} n+\frac{1}{2\left(1-\lambda_{4}\right)} n^{2}+\frac{\lambda_{4}^{n+3}}{\left(\lambda_{4}-1\right)^{3}}
$$

for $n \geq-3$. Besides, from (96) we have

$$
\lambda_{4}=a-3 .
$$

Corollary 4 Consider equation (17) with $a, b, c \in \mathbb{C}$ and $d \in \mathbb{C} \backslash\{0\}$. Assume that $\Delta=0$, $\Delta_{0}=0$, and $D \neq 0$. Then the following statements hold.

(a) If $3 a+2 b+c \neq 4$, then the general solution to equation (17) is given by formula (21), where sequence $\left(s_{n}\right)_{n \geq-3}$ is given by (101).

(b) If $a+b+c+d=1$ and $3 a+2 b+c=4$, then the general solution to equation (17) is given by formula (21), where sequence $\left(s_{n}\right)_{n \geq-3}$ is given by (102), whereas $\lambda_{4}$ is given by (103).

Case $\Delta=0, D=0$, and $\Delta_{0}=0$. By Lemma $2(\mathrm{e})$ we have that all the zeros to $P_{4}$ are equal to $a / 4$.

The general solution in this case is given by

$$
a_{n}=\left(c_{1}+c_{2} n+c_{3} n^{2}+c_{4} n^{3}\right)\left(\frac{a}{4}\right)^{n}, \quad n \geq-3
$$

From this and (20) it follows that $s_{0}=1=c_{1}$,

$$
\begin{aligned}
& c_{1}-c_{2}+c_{3}-c_{4}=0, \\
& c_{1}-2 c_{2}+4 c_{3}-8 c_{4}=0, \\
& c_{1}-3 c_{2}+9 c_{3}-27 c_{4}=0 .
\end{aligned}
$$


Solving the system, we get

$$
c_{1}=c_{3}=1, \quad c_{2}=\frac{11}{6}, \quad \text { and } \quad c_{4}=\frac{1}{6} .
$$

Using this in (104), after some calculation, we obtain

$$
s_{n}=\frac{(n+1)(n+2)(n+3)}{6}\left(\frac{a}{4}\right)^{n}
$$

for $n \geq-3$.

Corollary 5 Consider equation (17) with $a, b, c \in \mathbb{C}$ and $d \in \mathbb{C} \backslash\{0\}$. Assume that $\Delta=0$, $D=0$, and $\Delta_{0}=0$. Then the general solution to equation (17) is given by formula (21), where the sequence $\left(s_{n}\right)_{n \geq-3}$ is given by (105).

\section{Acknowledgements}

The study in the paper is a part of the investigations under the projects III 41025 and III 44006 by the Serbian Ministry of Education and Science.

\section{Funding}

Not applicable.

Availability of data and materials

Not applicable.

\section{Competing interests}

The author declares that he has no competing interests.

\section{Authors' contributions}

The author has contributed solely to the writing of this paper. He read and approved the manuscript.

\section{Author details}

${ }^{1}$ Mathematical Institute of the Serbian Academy of Sciences, Beograd, Serbia. ${ }^{2}$ Department of Medical Research, China Medical University Hospital, China Medical University, Taichung, Taiwan, Republic of China. ${ }^{3}$ Department of Computer Science and Information Engineering, Asia University, Taichung, Taiwan, Republic of China.

\section{Publisher's Note}

Springer Nature remains neutral with regard to jurisdictional claims in published maps and institutional affiliations.

Received: 16 July 2019 Accepted: 1 October 2019 Published online: 11 October 2019

\section{References}

1. de Moivre, A.: Miscellanea analytica de seriebus et quadraturis. J. Tonson \& J. Watts, London (1730) (in Latin)

2. Euler, L.: Introductio in Analysin Infinitorum. Tomus Primus, Lausannae (1748) (in Latin)

3. Boole, G.: A Treatsie on the Calculus of Finite Differences, 3rd edn. Macmillan \& Co., London (1880)

4. Brunacci, V.: Corso di Matematica Sublime, Tomo I, Calcolo delle Differenze Finite e sue Applicazioni. Presso Pietro Allegrini, Firenze (1804) (in Italian)

5. Fort, T.: Finite Differences and Difference Equations in the Real Domain. Clarendon, Oxford (1948)

6. Jordan, C.: Calculus of Finite Differences. Chelsea, New York (1956)

7. Levy, H., Lessman, F.: Finite Difference Equations. Dover, New York (1992)

8. Markov, A.A.: Ischislenie Konechnykh Raznostey, 2nd edn. Matezis, Odessa (1910) (in Russian)

9. Milne-Thomson, L.M.: The Calculus of Finite Differences. Macmillan \& Co., London (1933)

10. Mitrinović, D.S, Kečkić, J.D.: Methods for Calculating Finite Sums. Naučna Knjiga, Beograd (1984) (in Serbian)

11. Abel, N.H.: Mémoire sur les équations algébriques, où l'on démontre l'impossibilité de la résolution de l'équation générale du cinquième degré. In: Sylow, L., Lie, S. (eds.) Oeuvres Complètes de Niels Henrik Abel, vol. I, 2nd edn. pp. 28-33 (1881) [1824] (in French)

12. Berg, L., Stević, S.: On some systems of difference equations. Appl. Math. Comput. 218, 1713-1718 (2011)

13. Papaschinopoulos, G., Stefanidou, G.: Asymptotic behavior of the solutions of a class of rational difference equations. Int. J. Difference Equ. 5(2), 233-249 (2010)

14. Stević, S.: On the system of difference equations $x_{n}=c_{n} y_{n-3} /\left(a_{n}+b_{n} y_{n-1} x_{n-2} y_{n-3}\right), y_{n}=\gamma_{n} x_{n-3} /\left(\alpha_{n}+\beta_{n} x_{n-1} y_{n-2} x_{n-3}\right)$. Appl. Math. Comput. 219, 4755-4764 (2013)

15. Stević, S.: Solvable subclasses of a class of nonlinear second-order difference equations. Adv. Nonlinear Anal. 5(2), 147-165 (2016) 
16. Stević, S., Diblik, J., Iričanin, B., Šmarda, Z:: On some solvable difference equations and systems of difference equations. Abstr. Appl. Anal. 2012, Article ID 541761 (2012)

17. Stević, S., Diblik, J., Iričanin, B., Šmarda, Z:: On the difference equation $x_{n}=a_{n} x_{n-k} /\left(b_{n}+c_{n} x_{n-1} \cdots x_{n-k}\right)$. Abstr. Appl. Anal. 2012, Article ID 409237 (2012)

18. Stević, S.: Bounded and periodic solutions to the linear first-order difference equation on the integer domain. Adv. Differ. Equ. 2017, Article ID 283 (2017)

19. Stević, S.: Bounded solutions to nonhomogeneous linear second-order difference equations. Symmetry 9, Article ID $227(2017)$

20. Stević, S.: Existence of a unique bounded solution to a linear second order difference equation and the linear first order difference equation. Adv. Differ. Equ. 2017, Article ID 169 (2017)

21. Adamović, D.: Solution to problem 194. Mat. Vesn. 23, 236-242 (1971)

22. Brand, L.: A sequence defined by a difference equation. Am. Math. Mon. 62(7), 489-492 (1955)

23. Mitrinović, D.S., Adamović, D.D.: Sequences and Series. Naučna Knjiga, Beograd (1980) (in Serbian)

24. Richardson, C.H.: An Introduction to the Calculus of Finite Differences. Van Nostrand, Toronto (1954)

25. Stević, S.: Representation of solutions of bilinear difference equations in terms of generalized Fibonacci sequences. Electron. J. Qual. Theory Differ. Equ. 2014, Article ID 67 (2014)

26. Krechmar, V.A.: A Problem Book in Algebra. Mir, Moscow (1974)

27. Berezansky, L., Braverman, E.: On impulsive Beverton-Holt difference equations and their applications. J. Differ. Equ. Appl. 10(9), 851-868 (2004)

28. Stević, S.: Representations of solutions to linear and bilinear difference equations and systems of bilinear difference equations. Adv. Differ. Equ. 2018, Article ID 474 (2018)

29. Stević, S., Iričanin, B., Kosmala, W., Šmarda, Z.: Note on the bilinear difference equation with a delay. Math. Methods Appl. Sci. 41, 9349-9360 (2018)

30. Stević, S., Iričanin, B., Šmarda, Z:: On a symmetric bilinear system of difference equations. Appl. Math. Lett. 89, 15-21 (2019)

31. Alfred, B.U.: An Introduction to Fibonacci Discovery (1965)

32. Vorobiev, N.N.: Fibonacci Numbers. Birkhäuser, Basel (2002)

33. Stević, S.: First-order product-type systems of difference equations solvable in closed form. Electron. J. Differ. Equ. 2015, Article ID 308 (2015)

34. Stević, S.: Product-type system of difference equations of second-order solvable in closed form. Electron. J. Qual. Theory Differ. Equ. 2015, Article ID 56 (2015)

35. Stević, S.: Product-type system of difference equations with complex structure of solutions. Adv. Differ. Equ. 2017, Article ID 140 (2017)

36. Stević, S.: Solvable product-type system of difference equations whose associated polynomial is of the fourth order. Electron. J. Qual. Theory Differ. Equ. 2017, Article ID 13 (2017)

37. Stević, S.: Solvability of a product-type system of difference equations with six parameters. Adv. Nonlinear Anal. 8(1), 29-51 (2019)

38. Stević, S., Iričanin, B., Šmarda, Z:: On a product-type system of difference equations of second order solvable in closed form. J. Inequal. Appl. 2015, Article ID 327 (2015)

39. Stević, S., Iričanin, B., Šmarda, Z.: Two-dimensional product-type system of difference equations solvable in closed form. Adv. Differ. Equ. 2016, Article ID 253 (2016)

40. Mitrinović, D.S.: Matrices and Determinants. Naučna Knjiga, Beograd (1989) (in Serbian)

41. Papaschinopoulos, G., Schinas, C.J.: On the behavior of the solutions of a system of two nonlinear difference equations. Commun. Appl. Nonlinear Anal. 5(2), 47-59 (1998)

42. Papaschinopoulos, G., Schinas, C.J.: Invariants for systems of two nonlinear difference equations. Differ. Equ. Dyn. Syst. 7(2), 181-196 (1999)

43. Papaschinopoulos, G., Schinas, C.J.: Invariants and oscillation for systems of two nonlinear difference equations. Nonlinear Anal. TMA 46(7), 967-978 (2001)

44. Papaschinopoulos, G., Schinas, C.J., Stefanidou, G.: On a k-order system of Lyness-type difference equations. Adv. Differ. Equ. 2007, Article ID 31272 (2007)

45. Riordan, J: Combinatorial Identities. Wiley, New York (1968)

46. Schinas, C.: Invariants for difference equations and systems of difference equations of rational form. J. Math. Anal. Appl. 216, 164-179 (1997)

47. Schinas, C.: Invariants for some difference equations. J. Math. Anal. Appl. 212, 281-291 (1997)

48. Stević, S., Iričanin, B., Kosmala, W., Šmarda, Z.: Representation of solutions of a solvable nonlinear difference equation of second order. Electron. J. Qual. Theory Differ. Equ. 2018, Article ID 95 (2018)

49. Stević, S., Iričanin, B., Kosmala, W., Šmarda, Z.: On some classes of solvable systems of difference equations. Adv. Differ. Equ. 2019, Article ID 39 (2019)

50. Faddeyev, D.K.: Lectures on Algebra. Nauka, Moscow (1984) (in Russian)

51. Rees, E.L.: Graphical discussion of the roots of a quartic equation. Am. Math. Mon. 29(2), $51-55$ (1922) 